\title{
Marker-based development of wheat near-isogenic and substitution lines with high anthocyanin content in grains
}

\author{
Gordeeva E.I. ${ }^{1 *}$, Badaeva E.D. ${ }^{2}$, Adonina I.G. ${ }^{1}$, Khlestkina E.K. ${ }^{1,3}$, Shoeva O.Yu. ${ }^{1}$ \\ ${ }^{1}$ Institute of Cytology and Genetics, SB RAS, Novosibirsk, Russia \\ ${ }^{2}$ Vavilov Institute of General Genetics, RAS, Moscow, Russia \\ ${ }^{3}$ N.I. Vavilov All-Russian Institute of Plant Genetic Resources (VIR), St. Petersburg, Russia \\ *e-mail:elgordeeva@bionet.nsc.ru
}

Bread wheat (Triticum aestivum L., $2 n=6 x=42$, BBAADD) is one of the most important cereal crop. Today there is increasing interest in production of wheat with high anthocyanin content in grain, as a source of functional foods. Anthocyanins can be produced in wheat grain either in pericarp (under control of genes $P p$ ) or in aleurone $(B a)$. Previously, on the genetic background of Saratovskaya 29 cultivar we developed a set of near-isogenic lines having different combinations of anthocyanin biosynthesis regulatory genes $P p-A 1, P p-D 1$ and $P p 3$ as well as a blue-grained substitution line $\left(\mathrm{BC}_{7}\right.$ progeny) carrying the wheatgrass (Agropyron elongatum Host.) Ba genes. Chromosome C-banding, FISH and microsatellite analysis showed substitution of wheat chromosome 4D by Ag. elongatum chromosome 4Ag. The line was designed 'S29(Ag. elongatum 4Ag(4D))'. We constructed diagnostic markers for the dominant alleles of Pp3 (TaMyc1) inherited from purple wheat and $B a(T h M y c 4 E)$ inherited from $A g$. elongatum. The markers together with microsatellites linked to these genes were used for selection of plants with dominant alleles at the $P p$ and $B a$ loci. In the $\mathrm{F}_{2}$ progeny, obtained after crossing the blue- with the purple-grained lines, in addition to deep purple-grained plants with the dominant alleles of the $P p-1, P p 3$ and $B a(4 \mathrm{Ag} / 4 \mathrm{D})$ genes, blue-grained plants with hairy leaves ( $\mathrm{Hl}$ gene is localized in $4 \mathrm{BL}$ ) were found. FISH and molecular marker analysis of these plants confirmed the substitution $4 \mathrm{Ag} / 4 \mathrm{~B}$. The line was designed 'S29(Ag. elongatum 4Ag(4B))'. By crossing the new line with the purple-grained line, deep purple-grained plants with dominant alleles $P p-1, P p 3$ and $B a(4 \mathrm{Ag} / 4 \mathrm{~B})$ in one genome were obtained. The substitution and isogenic lines and the allele-specific markers designed in the study can be applied for accelerated obtaining wheat with high anthocyanins content in grains.

Acknowledgements: The study was partially supported by ICG project (0324-2019-0039) and RFBR (19-016-00140). 